\title{
Perfil químico e atividade antimicrobiana do óleo essencial de variedades de Psidium
}

\section{guajava L. (Myrtaceae)}

\author{
Chemical profile and antimicrobial activity of essential oil from Psidium guajava L. (Myrtaceae) \\ varieties
}

Perfil químico y actividad antimicrobiana del aceite esencial de variedades Psidium guajava $\mathbf{L}$. (Myrtaceae)

\author{
Janaína Kiara Miranda Trindade \\ ORCID: https://orcid.org/0000-0002-8441-2699 \\ Universidade Federal do Amazonas, Brasil \\ E-mail: kiarajana19@gmail.com \\ Ívina Thayna Miranda Trindade \\ ORCID: https://orcid.org/0000-0002-1184-2241 \\ Universidade Federal do Amazonas, Brasil \\ E-mail: ivinamiranda.17@gmail.com \\ Maxwell Adriano Abegg \\ ORCID: https://orcid.org/0000-0002-0328-1122 \\ Universidade Federal do Amazonas, Brasil \\ E-mail: maxabegg@gmail.com \\ Geone Maia Corrêa \\ ORCID: https://orcid.org/0000-0002-9458-8305 \\ Universidade Federal do Amazonas, Brasil \\ E-mail: geonemaia@ufam.edu.br \\ Dominique Fernandes de Moura do Carmo \\ ORCID: https://orcid.org/0000-0002-8835-1619 \\ Universidade Federal do Amazonas, Brasil \\ E-mail: dominiquefmc@ufam.edu.br
}

\begin{abstract}
Resumo
O gênero Psidium é pertencente à família Myrtaceae e compreende importantes espécies botânicas, destacando-se a goiabeira (Psidium guajava L.). O óleo essencial (OE) desta espécie já conduziu diversos trabalhos científicos devido a sua composição química e propriedades farmacológicas, destacando-se a sua ação antifúngica e atividades antimicrobianas. Neste trabalho, duas variedades de Psidium, Psidium guajava var. pomifera (Paluma) e Psidium guajava var. pyrifera (Kumagai), foram coletadas em diferentes localidades do município de Itacoatiara - AM, o OE das folhas foram avaliadas quanto à composição química e atividade antimicrobiana. A extração do óleo essencial foi realizada por hidrodestilação em aparelho do tipo Clevenger durante 4 horas, as análises quantitativas e qualitativas foram executadas por cromatografia gasosa acoplada à espectrometria de massa. As atividades antimicrobianas foram avaliadas utilizando o teste in vitro sobre a inibição do crescimento bacteriano de Escherichia coli (Migula 1895) Castellani e Chalmers 1919, Salmonella sp e Staphylococcus aureus Rosenbach 1884 e do crescimento micelial dos fitopatógenos Candida albicans (C.P. Robin) Berkhout 1923 e Candida parapsilosis (Ashford) Langeron \& Talice 1932. A composição química revelou a presença dos monoterpenos: D_limoneno, $\alpha$-pineno, 1,8 - cineol, e dos sequiterpenos: trans-nerolidol, trans-cariofileno, óxido de cariofileno e $\alpha$-Humuleno como constituintes majoritários nas amostras avaliadas. Em relação aos testes biológicos realizados foi possível observar atividade antimicrobiana em todas as cepas estudadas. Diante dos resultados, conclui-se que óleos essenciais destas variedades de $P$. guajava apresentam atividades promissoras e podem ser consideradas como nova fonte de compostos bioativos antimicrobianos.
\end{abstract}

Palavras-chave: Myrtaceae; Goiabeira; Potencial antimicrobiano.

\begin{abstract}
The genus Psidium belongs to the Myrtaceae family and comprises important botanical species, especially the guava tree (Psidium guajava L.). The essential oil (EO) of this species has already conducted several scientific studies due to its chemical composition and pharmacological properties, highlighting its antifungal action and antimicrobial activities. In this work, two varieties of Psidium, Psidium guajava var. pomifera (Paluma) and Psidium guajava var. pyrifera (Kumagai), were collected in different locations in the municipality of Itacoatiara - AM, the OE of the leaves were evaluated for chemical composition and antimicrobial activity. The extraction of essential oil was performed by
\end{abstract}


hydrodistillation in a Clevenger-type apparatus for 4 hours, quantitative and qualitative analyzes were performed by gas chromatography coupled with mass spectrometry. The antimicrobial activities were evaluated using the in vitro test on the inhibition of bacterial growth of Escherichia coli (Migula 1895) Castellani and Chalmers 1919, Salmonella sp and Staphylococcus aureus Rosenbach 1884 and mycelial growth of the phytopathogens Candida albicans (CP Robin) Berkhout 1923 and Candida parapsilosis (Ashford) Langeron \& Talice 1932. The chemical composition revealed the presence of the monoterpenes: D_limonene, $\alpha$-pinene, 1.8 - cineol, and the sequiterpenes: trans-nerolidol, trans-caryophyllene, caryophyllene oxide and $\alpha$-humulene as constituents majority in the evaluated samples. Regarding the biological tests performed, it was possible to observe antimicrobial activity in all strains studied. Based on the results, it is concluded that essential oils of these varieties of $P$. guajava have promising activities and can be considered as a new source of antimicrobial bioactive compounds.

Keywords: Myrtaceae; Guava; Antimicrobial potential.

\section{Resumen}

El género Psidium pertenece a la familia Myrtaceae y comprende importantes especies botánicas, especialmente el árbol de guayaba (Psidium guajava L.). El aceite esencial (AE) de esta especie ya ha realizado varios estudios científicos debido a su composición química y propiedades farmacológicas, destacando su acción antifúngica y antimicrobiana. En este trabajo, dos variedades de Psidium, Psidium guajava var. pomifera (Paluma) y Psidium guajava var. pyrifera (Kumagai), se recolectaron en diferentes localidades del municipio de Itacoatiara - AM, se evaluó la $\mathrm{OE}$ de las hojas en cuanto a composición química y actividad antimicrobiana. La extracción del aceite esencial se realizó mediante hidrodestilación en un aparato tipo Clevenger durante 4 horas, los análisis cuantitativos y cualitativos se realizaron mediante cromatografía de gases acoplada a espectrometría de masas. Las actividades antimicrobianas se evaluaron mediante el ensayo in vitro sobre la inhibición del crecimiento bacteriano de Escherichia coli (Migula, 1895) Castellani y Chalmers 1919, Salmonella sp y Staphylococcus aureus Rosenbach 1884 y el crecimiento micelial de los fitopatógenos Candida albicans (CP Robin) Berkhout 1923 y Candida parapsilosis (Ashford) Langeron \& Talice 1932. La composición química reveló la presencia de los monoterpenos: D_limoneno, $\alpha$-pineno, 1.8-cineol, y los sequiterpenos: trans-nerolidol, trans-cariofileno, óxido de cariofileno y $\alpha$-humuleno como principales constituyentes en las muestras evaluadas. En cuanto a las pruebas biológicas realizadas, se pudo observar actividad antimicrobiana en todas las cepas estudiadas. Con base en los resultados, se concluye que los aceites esenciales de estas variedades de P. guajava tienen actividades prometedoras y pueden considerarse como una nueva fuente de compuestos bioactivos antimicrobianos.

Palabras clave: Myrtaceae; Árbol de guayaba; Potencial antimicrobiano.

\section{Introdução}

Existe uma gama de relatos referente à ação inibitória de microrganismos quando tratados com extratos e óleos de plantas, dentre essas ações destacam-se a inibição frente à Escherichia coli, Salmonella sp., Staphylococcus aureus, Streptococcus mutans, Cryptococcus neoformans, Candida albicans e Candida tropicalis (Araújo, 2010; Garcia, 2018). Segundo Bastos (2016), esses agentes infecciosos tem sido uma das maiores causas de mortalidade, morbidade e preocupações médicas desde muito antes dos avanços tecnológicos. Essa problemática, causada principalmente nos ambientes hospitalares, é o que fundamenta a procura de novos fármacos antimicrobianos, principalmente de origens naturais, justamente por existirem apenas fármacos de origem sintéticos.

A família Myrtaceae Jussieu, destaca-se como a de maior uso fitoterápico e ainda é apontada como uma das maiores famílias botânicas, agrupando mais de 3600 espécies vegetais e por volta de 140 gêneros espalhadas pelo mundo, sendo que dentro desta família, a Psidium está entre as quatro (4) espécies com maior importância econômica do Brasil devido seus frutos com sabor exótico, e, além disso, possui em suas folhas abundância de constituintes, tais como fenólicos e óleo essencial (Durães et al., 2015).

Ainda segundo Durães (2015) Psidium guajava é a espécie quem tem ganhado maior destaque devido a grande utilidade de seus frutos com a produção de geleias e doces e no uso de suas folhas na medicina popular. Em estudos anteriores foram comprovadas as ações biológicas do extrato bruto das folhas de P. guajava no tratamento de diarreia, disenteria, doenças pulmonares e bronquite, outras propriedades também foram atestadas conferindo à espécie ações antiespasmódicas, 
antimicrobiana, anti-inflamatória, anticonvulsionante, analgésica, antidiabéticas, anti-hiperlipidêmica e antioxidante (Souza, 2015; Nascimento, 2017; Silva, 2019;).

Entre os estudos já realizados com o óleo essencial das folhas de Psidium guajava foram identificados os constituintes $\alpha$-humuleno, trans-cariofileno, limoneno, epóxido de humuleno II, óxido de cariofileno, $\alpha$-selineno, aromadendreno, selin-11en-4 $\alpha$-ol, 1,8-cineol, trans-nerolidol, $\beta$-bisaboleno, $\alpha$-pineno e $\beta$-sitosterol, (Alves et al., 2006) e atribuídas importantes atividades biológicas, destacando-se o potencial inseticida, antimicrobiano, antifúngico, antioxidante e larvicida (Souza, 2015; Silva, 2019). Segundo Ilha, et al. (2008) e Costa (2018), Psidium guajava L. (goiabeira) porta uma considerável quantia de açucares, pectinas e ácidos, além de compostos mais relevantes, tais como flavonoides, ácidos triterpenoides (oleanólico, ursólico, catecólico, guaiavólico, maslínico), sesquiterpenoides, e óleos essenciais (Haida et al., 2015).

A espécie Psidium guajava L, popularmente conhecida como goiabeira, é natural da região tropical do continente Americano, tendo como possível ponto central situado entre o Norte da América do Sul e Sul do México (Oliveira, 2013). É uma frutífera de copa aberta com uma altura de até $7 \mathrm{~m}$, no qual apresenta folhas oblongas, opostas, subcoriáceas, e aromáticas. Seus caules são quadrangulares, vigorosamente ou um tanto erguidos e frequentemente rugosos. Os frutos medem por volta de $10 \mathrm{~cm}$ de diâmetro na forma de baga, com sabor doce e ligeiramente aromático, podendo sua polpa apresentar coloração rosa ou avermelhada, amarela e branca (Campos, 2010).

\subsection{Psidium guajava var. pomifera (Paluma)}

A goiaba de polpa vermelha, variedade Paluma, foi produzida a partir da polimerização aberta das variedades Rubi e Supreme, por meio do programa de melhoramento genético da UNESP/FCAV de Jaboticabal, SP (Oliveira et al., 2012).

Oriunda da América tropical, provavelmente entre o Peru e o México (Pinho, 2015), é uma das espécies de maior importância econômica do Brasil, pois dispõe de características, como coloração avermelhada, casca lisa, polpa de textura firme, peso acima de $200 \mathrm{~g}$ e equilibrada acidez, e com propriedades de favoráveis processamentos, podendo ser utilizada, por exemplo, na produção de compotas, doces e sucos. (Torres et al., 2005).

Segundo Haida, et al. (2015) esta variedade detém quantidade significativa de vitamina C, com nível de 6 à 7 vezes superiores aos níveis das frutas cítricas, vitamina A e complexo B e presença de sais minerais, tais como ferro, fosforo e cálcio.

Seus resíduos desidratados mostraram a presença de carotenoides que são encarregados da coloração das frutas, ácido ascórbico, além de demostrar ação biológica (Moraes et al, 2015). Pinho (2015) menciona que o chá da goiaba vermelha tem ação antimicrobiana e terapêutica, e que o óleo essencial desta possui propriedades que podem ser empregadas no controle de pragas na agricultura e, portanto, é um importante agente bioinseticida.

\subsection{Psidium guajava var. pyrifera (Kumagai)}

A goiaba de polpa branca, variedade Kumagai, é provavelmente oriunda do cruzamento da goiaba Australiana com a goiaba IAC-4, em Campinas- SP (Ponzo, 2014). Esta dispõe de características como casca lisa, coloração de polpa branca, formato oblongo ou redondo, com peso que varia de $300 \mathrm{~g}$ á $400 \mathrm{~g}$, e ainda possui uma boa resistência ao ser transportada (Gonzaga Neto, 2007).

As duas variedades mais comuns espécie são a Psidium guajava var pomifera (goiaba vermelha) e a Psidium guajava var pyrifera (goiaba branca) (Pinho, 2015), no entanto, quando se fala da atividade biológica de seus óleos, a literatura dispõe de poucas informações, principalmente da goiaba branca.

De acordo com Lewinsohn, et al. (1998), apesar do avanço no conhecimento das propriedades biológicas e farmacológicas dos óleos vegetais, sabe-se pouco sobre a morfologia das estruturas celulares ou órgãos envolvidos na produção nos vegetais. Com isso, o presente trabalho busca caracterizar a composição química do óleo essencial das folhas de 
espécies de Psidium guajava, bem como avaliar o potencial antimicrobiano em Escherichia coli, Staphylococcus, Salmonella, Candida albicans e Candida parapsilosis, além de realizar a caracterização morfoanatômica das partes aéreas das variedades da espécie.

\section{Materiais e métodos}

\subsection{Coleta e identificação do material vegetal}

As folhas de Psidium guajava foram coletadas no mês de novembro de 2019 em diferentes localidades do município de Itacoatiara-AM. Foram selecionadas as variedades Psidium guajava var. pomifera (goiaba vermelha) e Psidium guajava var. pyrifera (goiaba branca), as exsicatas foram preparadas e depositadas no Herbário (HERBIT) da Universidade do Estado do Amazonas (UEA), obtendo o número de registro 0066 para a variedade branca e 0065 para variedade vermelha.

\subsection{Extração do óleo essencial}

Os óleos essenciais das espécies foram obtidos pelo método de hidrodestilação por arraste a vapor em aparelho modificado de Clevenger, utilizando-se 584,1g (Psidium guajava var. pomifera) e 977,49g (Psidium guajava var. pyrifera) de folhas frescas. Os materiais vegetais foram submetidos a aquecimento em contato direto com $6 \mathrm{~L}$ de água destilada a $100^{\circ} \mathrm{C}$ até atingir a fervura, sendo reduzindo posteriormente a $40^{\circ} \mathrm{C}$, com tempo de extração de 4 horas ininterruptas, após o inicio da ebulição. Em seguida as amostras extraídas foram direcionadas a centrifuga (modelo 80-2B, centrifuge 2013) para posterior separação óleo/água, sendo depositadas em microtubos e armazenadas sob refrigeração a $-10{ }^{\circ} \mathrm{C}$ para evitar perdas de constituintes voláteis.

\subsection{Espectrometria de massas (MS)}

A análise qualitativa e quantitativa dos constituintes dos óleos essenciais, extraídos das folhas, foi realizada por Cromatografia Gasosa acoplada à Espectrometria de Massa (GC-MS) (Shimadzu GCMS-QP2010 SE), a quantificação dos constituintes identificados foi estimada através da normalização da aérea (\%) dos picos no cromatograma organizados em ordem de diluição (Adams, 2007).

Para essas análises, as seguintes condições foram empregadas: o gás arraste utilizado foi o He para os dois detectores com fluxo e velocidade linear de 1,30 mL. $\mathrm{min}^{-1}$ e 41,4,9 cm/seg (GC-MS), a temperatura do injetor foi de $230{ }^{\circ} \mathrm{C}$ na razão split de 1:30; coluna capilar de sílica fundida (30 m x 0,25 mm); fase estacionária Rtx-5MS (0,25 $\mu \mathrm{m}$ de espessura do filme); a temperatura do forno teve a seguinte programação: temperatura inicial de $50{ }^{\circ} \mathrm{C}$, a qual permaneceu por 3 minutos e em seguida a temperatura foi aumentada gradativamente a $3{ }^{\circ} \mathrm{C} \cdot \mathrm{min}^{-1}$ até atingir $180^{\circ} \mathrm{C}$, em que permaneceu por 10 minutos, tendo um tempo total de análise de $63,33 \mathrm{~min}$; a temperatura utilizada no detector MS foi de $240{ }^{\circ} \mathrm{C}$.

As amostras utilizadas foram retiradas dos vials em um volume de $1 \mu \mathrm{L}$ de uma solução de $3 \%$ de óleo essencial dissolvido em hexano com DMA 0,1 mol.L-1 (padrão externo para controle de reprodutibilidade). As análises por GC-MS foram realizadas em um equipamento por impacto eletrônico com energia de impacto de $70 \mathrm{eV}$; velocidade de varredura de 1000; intervalo de varredura de 0,50 fragmentos. $\mathrm{seg}^{-1}$ e fragmentos detectados de 10 a 700 (m/z). A identificação dos componentes dos óleos essenciais foi realizada pela comparação dos espectros de massas obtidos com os disponíveis no banco de dados da espectroteca (Wiley 7, NIST 05 e NIST 05s) e pelos índices de retenção de Kovats (IK). Para o cálculo dos IK, foi utilizada uma mistura de alcanos saturados $\mathrm{C}_{7}-\mathrm{C}_{40}$ (Supelco-USA) e o tempo de retenção ajustado de cada composto, obtidos através do GC-FID. Em seguida, os valores calculados para cada composto foram comparados com os da literatura (Adams, 2007; Nist, 2011; El-Sayed, 2016). 


\subsection{Atividade antimicrobiana}

O ensaio de avaliação antimicrobiana foi efetuado empregando o teste de microdiluição em microplacas para a análise do potencial antibacteriano e antifúngico dos óleos essências das espécies de $P$. guajava adaptado de Noumi, et al. (2018).

\subsection{Cultivos e preparação das suspensões das cepas}

As cepas bacterianas e fúngicas foram cultivadas em caldo Muller Hilton por $24 \mathrm{~h}$ a $34^{\circ} \mathrm{C}$. Em seguida foi verificada por meio de espectrofotômetro a determinação das concentrações desses microrganismos. A concentração padronizada para as bactérias foi de 0,02 colônias $/ \mathrm{mL}$ e paras os fungos 0,05 colônias $/ \mathrm{mL}$. Para isso foi retirado com auxílio de uma alça estéreo certa quantidade das cepas bacterianas e fúngicas e depositadas em $5 \mathrm{~mL}$ do caldo estéreo LB para as bactérias e $5 \mathrm{~mL}$ do caldo YPD para os fungos, e posteriormente levado ao espectrofotômetro.

\subsection{Preparação da amostra estoque dos óleos}

Inicialmente preparou-se a solução de DMSO $10 \%$ a partir de $90 \mathrm{~mL}$ de água destilada estéreo acrescido de $10 \mathrm{~mL}$ de dimetil sulfóxido. Em seguida, foram estocadas duas amostras em concentração de $100 \mathrm{mg} / \mathrm{mL}$ para cada variedade da espécie vegetal a ser estudado.

\subsection{Ensaio de microdiluição para bactérias}

O ensaio de determinação do CIM (concentração inibitória mínima) antibacteriano foi realizado em duplicata, onde as bactérias utilizadas foram E. coli, S. aureus e Salmonella sp. e como padrão de referência (controle positivo) o antibacteriano Cloranfenicol, e como controle negativo o DMSO. Os 4 poços correspondentes de cada espécie vegetal foram adicionados 90 $\mu \mathrm{L}$ do caldo LB acrescido com $100 \mu \mathrm{L}$ da solução estoque de óleo no primeiro poço para a realização da diluição seriada de 50 a $0,39 \mu \mathrm{L} / \mathrm{mL}$. Sendo que para os controles positivos foram adicionados $90 \mu \mathrm{L}$ do caldo $\mathrm{LB}$ acrescido com $100 \mu \mathrm{L}$ do Cloranfenicol e para o negativo, foram $100 \mu \mathrm{L}$ do caldo LB acrescido com $100 \mu \mathrm{L}$ do DMSO 10\% nos primeiros poços respectivos de cada controle para a realização da diluição seriada. A última diluição (linha H) foi descartada. Subsequentemente foram adicionados $10 \mu \mathrm{L}$ das suspensões dos microrganismos em cada poço e encubadas por $24 \mathrm{~h}$ à $37^{\circ} \mathrm{C}$.

\subsection{Ensaio de microdiluição para fungos}

O ensaio de determinação do CIM (concentração inibitória mínima) antifúngica foi realizado em duplicata, onde os fungos utilizados foram C. albicans e C. parapsilosis e como padrão de referência (controle positivo) o Metanol e como controle negativo DMSO 10\%. Os 4 poços correspondentes de cada espécie vegetal foram adicionados $90 \mu \mathrm{L}$ do caldo YPD acrescido com $100 \mu \mathrm{L}$ da solução estoque de óleo no primeiro poço para a realização da diluição seriada de 50 a $0,39 \mu \mathrm{g} / \mathrm{mL}$. A última diluição (linha $\mathrm{H}$ ) foi descartada. Sendo que para os controles positivos foram adicionados $90 \mu \mathrm{L}$ do caldo YPD com $100 \mu \mathrm{L}$ do Metanol e para o negativo, foi $100 \mu \mathrm{L}$ do caldo YPD acrescido com $100 \mu \mathrm{L}$ do DMSO 10\% nos primeiros poços para a realização da diluição seriada. Subsequentemente foram adicionados $10 \mu \mathrm{L}$ das suspensões dos microrganismos nos poços e encubadas por $48 \mathrm{~h}$ à $37^{\circ} \mathrm{C}$. Para ambos os ensaios, foi utilizada a resazurina $(5 \mu \mathrm{g} / \mathrm{mL})$ como parâmetro visual para leitura das microplacas após o período de incubação dos microrganismos. Foram pesados $0,0015 \mathrm{~g}$ da resazurina e diluída em $10 \mathrm{~mL}$ de água destilada estéreo e $0,0060 \mathrm{~g}$ e diluída em $40 \mathrm{~mL}$ de água destilada estéreo. Posteriormente foram adicionados $10 \mu \mathrm{L}$ dessa solução nas microplacas e novamente foram encubadas por $2 \mathrm{~h}$. 


\section{Resultados e discussões}

\subsection{Extração e propriedades físicas dos óleos essenciais}

A hidrodestilação das folhas das duas variedades de $P$. guajava forneceu óleos levemente viscosos, de coloração amarelo claro e odor característico. A variedade vermelha, codificada como Pgvpom, apresentou um rendimento de 0,147\% e a branca, codificada como Pgvpyr, foi de 0,702\%.

\subsection{Cromatografia Gasosa Acoplada à Espectrometria de Massas (CG-MS)}

As análises por GC/EM permitiram a identificação de monoterpenos, sesquiterpenos oxigenados e hidrogenados nas amostras analisadas. No óleo essencial das folhas de Pgvpom foram identificados 43 constituintes, correspondente a 76,78\% da sua composição, sendo o óxido de Cariofileno (29,10\%), $\alpha$-Pineno $(18,61 \%)$ e D-limoneno (12,69\%) os componentes majoritários (Tabela 1).

No óleo essencial das folhas de Pgvpyr foram identificados 35 constituintes, correspondente a 67,30\% de composição, sendo o monoterpeno limoneno (18,71\%) e o sesquiterpeno $\alpha$-Humuleno (16,07\%) os componentes majoritários (Tabela 2). Nos estudos realizados por Lima, et al. (2011) com OE de P. guajava, foi possível identificar o 1,8-Cineol (48,8\%) como o constituinte principal, em outro estudo, realizado por Satyal, et al. (2015) foram identificados os sesquiterpenos (E)-Nerolidol $(35,6 \%)$ e $(E)$-Cariofileno (15,8\%) como os constituintes majoritários. Anteriormente, Pereira (2010) avaliando a composição do óleo essencial das folhas da espécie Psidium verificou a presença do Limoneno, $\alpha$-Pineno, $\beta$-Pineno, $\gamma$-Terpineno, $\alpha$ Copaeno, óxido de cariofileno e 1,8-cineol, corroborando com os resultados obtidos no presente trabalho.

Tabela 1. Composição química dos principais constituintes do óleo essencial de Pgvpom.

\begin{tabular}{|c|c|c|c|c|}
\hline Composto & Área (\%) & TR & $\mathbf{I K}_{\text {cal }}$ & $\mathbf{I K}_{\mathrm{tab}}$ \\
\hline$\alpha$-Pineno & 18,61 & 6265 & 933 & 0932 \\
\hline Canfeno & 0,09 & 6685 & 946 & 0946 \\
\hline D-Limoneno & 12,69 & 9430 & 1035 & 1024 \\
\hline 1,8-Cineol & 2,72 & 9495 & 1038 & 1026 \\
\hline Cis-Verbenol & 2,72 & 12515 & 1136 & 1137 \\
\hline Óxido de cis-Limoneno & 0,19 & 13670 & 1173 & 1132 \\
\hline Cis-p-mentha-2,8-dien-1-ol & 0,32 & 13765 & 1176 & 1133 \\
\hline Pinocarvona & 0,16 & 14890 & 1213 & 1160 \\
\hline Endo-Borneol & 0,15 & 15060 & 1218 & 1165 \\
\hline Mentha-1(7),8-dien-2-ol< Trans-p $>$ & 0,29 & 16050 & 1250 & 1287 \\
\hline Mirtenol & 0,26 & 16435 & 1263 & 1194 \\
\hline Trans-Carveol & 1,27 & 17450 & 1296 & 1215 \\
\hline Mentha-1(7),8-dien-2-ol< cis-p $>$ & 0,07 & 17840 & 1309 & 1127 \\
\hline Cis-Carveol & 0,34 & 17985 & 1313 & 1226 \\
\hline (1R,2R,3S,5R)-(-)-2,3-Pinanediol & 0,17 & 19850 & 1374 & 1318 \\
\hline Trans-cariofileno & 0,36 & 25875 & 1417 & 1417 \\
\hline$\gamma$-Muuroleno & 0,14 & 28265 & 1476 & 1478 \\
\hline Óxido de Cariofileno & 29,10 & 31230 & 1549 & 1482 \\
\hline
\end{tabular}


Research, Society and Development, v. 10, n. 10, e211101018794, 2021

(CC BY 4.0) | ISSN 2525-3409 | DOI: http://dx.doi.org/10.33448/rsd-v10i10.18794

\begin{tabular}{cccccc}
\hline Trans - Nerolidol & 1,18 & 31785 & 1562 & 1561 \\
Isocariofileno & 0,84 & 32145 & 1571 & NI \\
Epóxido de Humuleno II & 2,97 & 33425 & 1603 & 1608 \\
Cadin-4-en-10-ol & 0,32 & 34700 & 1634 & NI \\
Epi- $\alpha$-Cadinol & 1,77 & 34860 & 1638 & 1638 \\
$\beta$-Eudesmol & 0,45 & 34970 & 1641 & 1649 \\
\hline
\end{tabular}

*TR: Tempo de Retenção; IK cal: Índice de Kovats calculado; IK tab: Índice de Kovats tabelado; NI: Não Identificado. Fonte: Autores.

Tabela 2. Composição química dos principais constituintes do óleo essencial de Pgvpyr.

\begin{tabular}{|c|c|c|c|c|}
\hline Composto & Área (\%) & TR & $\mathbf{I K}_{\mathrm{cal}}$ & $\overline{I_{\text {tab }}}$ \\
\hline$\alpha$ - Tujeno & 0,01 & 6060 & 926 & 0924 \\
\hline$\alpha$-Pineno & 0,61 & 6245 & 932 & 0932 \\
\hline Benzaldeído & 0,15 & 7115 & 960 & 0952 \\
\hline$\beta$-Pineno & 0,04 & 7570 & 975 & 0974 \\
\hline$\beta$-Mirceno & 0,29 & 8075 & 991 & 0988 \\
\hline D-Limoneno & 18,77 & 9575 & 1040 & 1024 \\
\hline 1,8-Cineol & 6,25 & 9530 & 1039 & 1026 \\
\hline Trans- $\beta$-Ocimeno & 0,17 & 9810 & 1048 & 1044 \\
\hline$\beta$-Ocimeno & 0,05 & 10230 & 1061 & 1044 \\
\hline$\gamma$-Terpineno & 0,16 & 10595 & 1073 & 1054 \\
\hline Mentha-1(7),8-dien-2-ol $<$ trans-p $>$ & 0,12 & 16050 & 1250 & 1287 \\
\hline$\alpha$-Copaeno & 0,05 & 24090 & 1373 & 1374 \\
\hline Trans-Cariofileno & 8,45 & 25930 & 1418 & 1417 \\
\hline$\alpha$-Humuleno & 16,07 & 27365 & 1454 & 1452 \\
\hline$\beta$-Selineno & 4,03 & 28640 & 1485 & 1489 \\
\hline$\alpha$-Selineno & 3,17 & 29010 & 1494 & 1498 \\
\hline$\beta$-Bisaboleno & 0,04 & 29595 & 1509 & 1505 \\
\hline$\alpha$-Maalieno & 0,06 & 29870 & 1515 & NI \\
\hline Nerolodol B (Cis ou Trans) & 1,96 & 31785 & 1562 & 1561 \\
\hline Álcool Cariofileno & 0,20 & 31915 & 1566 & NI \\
\hline Óxido de Cariofileno & 3,65 & 32425 & 1578 & 1482 \\
\hline Epóxido de Humuleno II & 5,19 & 33450 & 1603 & 1608 \\
\hline Intermedeol & 0,77 & 33595 & 1607 & 1665 \\
\hline Mentha-1 (7),8-dien-2-ol< Cis-p > & 9,23 & 34395 & 1846 & 1227 \\
\hline$\beta$-Eudesmol & 0,10 & 34985 & 1641 & 1649 \\
\hline Intermedeol & 5,99 & 35195 & 1646 & 1665 \\
\hline
\end{tabular}

*TR: Tempo de Retenção; IK cal: Índice de Kovats calculado; IK tab: Índice de Kovats tabelado; NI: Não Identificado. Fonte: Autores. 


\subsection{Ensaio de Microdiluição}

\subsubsection{Atividade biológica}

Os valores de concentração inibitória determinadas para Pgvpom foram de $25 \mu \mathrm{g} / \mathrm{mL}$ contra Salmonella $s p$ e $S$. aureus, sendo inativo para a E. coli. De acordo com Holetz, et al. (2002) atividade antibacteriana é considerada boa quando o valor de CIM está abaixo de $100 \mu \mathrm{g} / \mathrm{mL}$, moderada quanto os valores estão entre 100 a $500 \mu \mathrm{g} / \mathrm{mL}$, fraca quando os valores estão entre 500 e $1000 \mu \mathrm{g} / \mathrm{mL}$ e inativa quando o valor encontrado for maior de $1000 \mu \mathrm{g} / \mathrm{mL}$, portanto, os resultados encontrados mostraram que a amostra de óleo essencial exibiu alta atividade antibacteriana contra Salmonella sp e $S$. aureus sugerindo uma atividade promissora contra bactérias fitopatogênicas. Souza e colaboradores (2018) comprovaram o potencial antibacteriano dos óleos essenciais das folhas frescas de P. guajava contra Streptococcus salivarius, S. mutans, S. mitis, $S$. sanguinis e S. sobrinus, para os valores encontrados foi possível classificar as atividades entre boa e moderada, além disso, o óleo essencial de P. guajava já foi descrito como promissor contra o fitopatógeno Sclerotinia sclerotiorum, causador de danos à agricultura (Silva et al., 2018).

O óleo essencial da amostra Pgvpyr exibiu alta atividade antibacteriana frente à espécie $E$. coli com valor de CIM = $25 \mu \mathrm{g} / \mathrm{mL}$.

Em um estudo realizado por Leite, et al. (2007) foi observada atividade antibacteriana do óleo essencial das folhas de Picea excelsa, na amostra foram identificados os monoterpenos $\alpha$ - pineno, $\beta$-pineno, limoneno e campeno como constituintes majoritários, indicando que a presença destes constituintes favoreceu a atividade antibacteriana observada. A atividade antibacteriana pode ser explicada pela natureza lipofílica dos monterpenos, acredita-se que estes constituintes causam dano direto na membrana celular, afetando a manutenção do pH celular e o equilíbrio dos íons inorgânicos (Oliveira et al., 2016).

Em outros trabalhos já foram reportadas a atividade antibacteriana de sesquiterpenos como óxido de cariofileno, trans - cariofileno e $\alpha$-humuleno (Moreira et al., 2014; Souza, 2015; Silva et al., 2016; Silva, 2019).

Estas informações justificam as atividades observadas nos óleos essenciais avaliados neste trabalho, considerando que os compostos majoritários identificados foram os monoterpenos $\alpha$ - Pineno e Limoneno, e os sesquiterpenos $\alpha$-humuleno e óxido de cariofileno.

Os indicativos de atividade biológica das duas variedades de óleos corroboram com os resultados publicados por Pereira (2010), mesmo sem este mencionar que variedade de $P$. guajava estudou. Outra pesquisa, realizada Luchesi (2017), foi identificada atividade antibacteriana de $P$. guajava frente a espécies $S$. aureus.

Os resultados das avaliações dos efeitos in vitro dos óleos essenciais de Pgvpom sobre o crescimento de C. albicans e C. parapsilosis foram de $12,5 \mu \mathrm{g} / \mathrm{mL}$ e $25 \mu \mathrm{g} / \mathrm{mL}$, respectivamente. Para a amostra Pgvpyr, os resultados apresentaram os mesmos valores de concentração de inibição mínima, que foram de $25 \mu \mathrm{g} / \mathrm{mL}$ (Tabela 3).

Tabela 3. Atividades antimicrobianas de (valores de CIM em $\mu \mathrm{g} / \mathrm{mL}$ ) de Pgvpom and Pgvpyr.

\begin{tabular}{c|c|c|c|c|c|c}
\hline Variedades da espécie $P$. & Amostras de oleo & \multicolumn{3}{|c|}{ Cepas bacterianas } & \multicolumn{2}{c}{ Cepas fúngicas } \\
\cline { 3 - 6 } guajava & essencial & SA & Salmonella $s p$ & EC & CA & CP \\
\hline $\begin{array}{c}\text { Psidium guajava } \text { var. } \\
\text { pomifera }\end{array}$ & Pgvpom & 25 & 25 & $>1000$ & 12,5 & 25 \\
\hline $\begin{array}{c}\text { Psidium guajava } \text { var. } \\
\text { pyrifera }\end{array}$ & Pgvpyr & $>1000$ & $>1000$ & 25 & 25 & 25 \\
\hline
\end{tabular}

*Pgvpom: Psidium guajava var. pomifera; Pgpvr: Psidium guajava var. pyrifera; SA: Staphylococcus aureus (ATCC 25923); Salmonella sp (cepa de laboratório); EC: Escherichia coli (ATCC 25922); CA: Candida albicans (ATCC 10231); CP: Candida parapsilosis (ATCC 22019). Fonte: Autores. 
Os resultados obtidos podem ser justificados pela composição química dos óleos, na amostra Pgvpom foi possível encontrar importantes metabólitos com potencial fungicida, destacando-se monoterpenos como D_limoneno (12.69\%), $\alpha$ pineno (18,61\%) e 1,8 - cineol (2,72\%) (Silva et al., 2018); sesquiterpenos como trans-nerolidol (1,18\%), trans-cariofileno $(0,36 \%)$ e óxido de cariofileno $(29,10 \%)$, além de outros compostos em baixas concentrações, que não podem ser descartados da composição (Arain et al., 2017; Hanif et al., 2018; Borah et al., 2019). Na amostra Pgvpyr foram identificados os monoterpenos D-Limoneno na concentração de $18,77 \%$ e 1,8-Cineol com 6,25\%, e os sesquiterpenos $\alpha$-Humuleno com rendimento de $16,07 \%$ e trans-cariofileno com $8,45 \%$ de concentração. Estes constituintes apresentam ação fungicida comprovada na literatura (Silva et al., 2018).

Em alguns trabalhos são citados a ação antifúngica das folhas de P.guajava, por exemplo, Hanif, et al. (2018) comprovaram a ação do óleo essencial das folhas da espécie supracitada contra os fungos Aspergillus niger, Aspergillus flavus, Fusarium solani e Rhizopus solani, no mesmo ano Silva e colaboradores identificaram a atividade antifúngica dos óleos contra S. sclerotiorum, obtendo-se mais de 90\% inibição. Cárceres, et al. (1991) comprovaram atividade antimicótica do extrato hidroalcoólico das folhas de P.guajava contra C. albicans, C. krusei, C. parapsilosis e C. stellatoidea.

Não há nenhum relato sobre a ação antibacteriana dos óleos essenciais das folhas das duas variedades de goiabeira contra Salmonella e E.coli e antifúngica contra C. albicans e C. parapsilosis. Os resultados obtidos sugerem que a espécie pode ser considerada como uma nova fonte de compostos bioativos.

\section{Conclusões}

O estudo sobre a composição química dos óleos essenciais das duas variedades de goiabeiras possibilitou a identificação de monoterpenos e sesquiterpenos como constituintes majoritários, infere-se que a presença dos monoterpenos D_limoneno, $\alpha$-pineno, 1,8 - cineol, e os sequiterpenos trans-nerolidol, trans-cariofileno, óxido de cariofileno e $\alpha$-Humuleno contribuíram para expressiva atividade antimicrobiana observada. A variedade $P$. guajava var. pomifera apresentou atividade antbibacteriana e antifúngica sobre as leveduras de Salmonella sp., S. aureus, C. albicans e $C$. parapsilosis, a variedade $P$. guajava var. pyrifera apresentou elevada atividade contra E. coli, C. albicans e C. parapsilosis. Todas as amostras que foram consideradas ativas quanto ao potencial antibacteriano e antifúgico apresentaram concentrações inibitórias inferiores a 50 $\mu \mathrm{g} / \mathrm{mL}$.

Há poucos relatos sobre as atividades biológicas, principalmente antibacteriana e antifúngica, do óleo essencial da $P$. guajava, assim este trabalho, além de contribuir com os conhecimentos químicos desta espécie, também contribuiu para avaliação da atividade biológica dos óleos essenciais da espécie quando aplicados em microrganismos. Estes resultados fornecem importantes informações de $P$. guajava var. pomifera e $P$. guajava var. pyrifera como fontes promissores de agentes naturais antimicrobianos e, neste contexto, justificam futuros estudos (in vitro e in vivo) dos seus constituintes químicos como coadjuvantes no controle de microorganismos.

\section{Agradecimentos}

Os autores agradecem a Fundação de Amparo à Pesquisa do Estado do Amazonas - FAPEAM (processo nº . 001/2021 - Mulheres na Ciência) pelo auxílio financeiro. 


\section{Referências}

Adams, R. P. (2007). Identification of Essential Oils Components by Gas Chromatography/Mass Spectroscopy. Allured Publishing Corporation, p. 804.

Alves, P. M., leite, P. H. A. S., Pereira, J. V., Pereira, L. F., Pereira, M. S. V., Higino, J. S. \& Lima, E. O. (2006). Atividade antifúngica do extrato de Psidium guajava Linn. (goiabeira) sobre leveduras do gênero Candida da cavidade oral: uma avaliação in vitro. Revista Brasileira de Farmacognosia, 16 (2), 193-194.

Arain, A., Sherazi, S. T. H. S., Mahesar, S. A. \& Sirajuddin. (2017). Spectroscopic and chromatographic evaluation of solvent extracted guava seed oil. International Journal of Food Properties, 20 (1), 556-563.

Araújo, E. R. S. (2010). Avaliação da biocompatibilidade e determinação da atividade antimicrobiana de Psidium Guineense Swartz. Dissertação (Mestrado: Programa de Pós - Graduação em Patologia) - Universidade Federal de Pernambuco, Recife.

Bastos, S.I. (2016). Avaliação da atividade antibacteriana, antifúngica e antimalárica de extratos, frações e composto obtidos de plantas da região amazônica. Dissertação (Mestrado em Saúde, Sociedade e Endemias na Amazônia) - Universidade Federal do Amazonas, Manaus.

Borah, A., Pandey, S. K., Saikat Haldar \& Mohan Lai. (2019). Chemical Composition of Leaf Essential Oil of Psidium guajava L. from North East India Journal of Essential Oil Bearing Plants, 1, 248-453.

Campos, L. Z. O. (2010). Etnobotânica do gênero Psidium L. (Myrtaceae) no Cerrado brasileiro. Dissertação (Mestrado em Botânica) - Universidade de Brasília, Brasília, 2p.

Caceres, A., Alvarez, A. V., Ovando, A.E. \& Samayoa, B. E. (1991). Plants used in Guatemala for the treatment of respiratory disease..Screening of 68 plants against gram-positive bacteria. Journal Ethnopharmacology, 31, 193-208.

Costa, W. S. (2018). Perfil de ácidos graxos da semente de goiaba (Psidium guajava L.), monografia (Graduação em Licenciatura em Química) - Instituto Federal de Educação, Ciências e Tecnologia do Piauí, Paraíba, 20p.

Donato, A. M. \& Morretes, B. L. (2005). Estudo anatômico das folhas de Psidium widgrenianum Berg. (Myrtaceae), uma potencial espécie medicinal. Rev. Bras. Farm, 86 (2), 65-70.

Durães, E. B., De Paula, J. A. M. \& Naves, P. L. F. (2015). Gênero Psidium: aspectos botânicos, composição química e potencial farmacológico. Revista Processos químicos, 9 (17), 34-35.

El-sayed, A. M. (2016). The Pherobase Database of Pheromones and Semiochemicals. <http://www.pherobase.com>.

Garcia, M. O. (2018). Atividade antimicrobiana de extratos e óleos essenciais de araçá (Psidium cattleianum S.) e pitanga (Eugenia uniflora L.) sobre patógenos de origem alimentar. Dissertação (Mestrado em Ciência e Tecnologia de Alimentos) - Universidade Federal de Pelotas, Faculdade de Agronomia Eliseu Maciel, Pelotas, RS.

Gonzaga, N. L. (2007). Produção da goiaba. In: 14ª Semana Internacional Da Fruticultura, Floricultura E Agroindústria - Frutal, Fortaleza, 24-29.

Haida, K. S., Haas, Mello, S. A. D., Haida, K. S., Abrão, R. M. \& Sahd, R. (2015). Compostos fenólicos e atividade antioxidante de goiaba (Psidium guajava L.) fresca e congelada. Revista Fitos, 9 (1), 38-39.

Hanif, M. U., Hussain, A. I., Chatha, S. A. S. \& Kamal, G. M. (2018). Variation in composition and bioactivities of essential oil from leaves of two different cultivars of Psidium guajava L. Journal of Essential Oil Bearing Plants, 21, 65-76.

Holetz, F.B., Pessini, G. L, Sanches, N. R., Cortez, D. A. G., Nakamura, C. V. \& Dias Filho, B.P. (2002). Screening of some plants used in the Brazilian folk medicine for the treatment of infections diseases. Memorias do Instituto Oswaldo Cruz, 97 (7), 1027-1031.

Ilha, S. M., Migliato.,K. F., Vellosa, J. C. R., Sacramento, L. V. S., Pietro, R. C. L. R., Isaac, V. L B, Brunetti, I. L., Corrêa, M. A. \& Salgado, H. R. N. (2008). Estudo fitoquímico de goiaba (Psidium guajava L.) com potencial antioxidante para desenvolvimento de formulação fitocosmética. Revista Brasileira de farmacognosia, 18 (3), 387-393.

Leite, A.M., Lima, E. O., Souza, E. L., Diniz, M. F. F. M., Trajano, V. N. \& Medeiros, I. A. (2007). Inhibitory effect of $\beta$-pinene, $\alpha$-pinene and eugenol on the growth of potential infectious endocarditis causing Gram-positive bacteria. Revista Brasileira de Ciências Farmacêuticas, 43 (1), $121-126$.

Leite, N. F. (2014). Composição fenólica e avaliação da atividade antioxidante e citoprotetora dos extratos de Psidium guajava L. var. pyrifera e Psidium guajava L. var. pomifera. Caderno de Ciência e Cultura, 13 (1), 10-13.

Lima, M. A. A., Oliveira, F. F. M., Gomes, G. A., Lavor, P. L., Santiago, G. M.P., Nagão-Dias, A. T., Arriaga, A. M. C., Lemos, T. L. G. \& Carvalho, M. G. (2011). Evaluantion of larvicida activity of the essential oils of plants species from Brazil against Aedes aegypti (Diptera: Culicidae). African Journal of Biotechnology, 10 (55), 11716-11720.

Luchesi, L. A. (2017). Atividade antibacteriana, antifúngica e antioxidante de óleos essenciais. Dissertação (Mestrado em Agroecossistemas) - Universidade Tecnologica do Paraná, Dois vizinhos, $88 \mathrm{f}$.

Moraes, F. P., Silva, E. S. da, Rocha, P. M., Silva, A. S. Da. \& Correia, R. T. P. (2015). Avaliação dos compostos bioativos presentes no resíduo de goiaba vermelha (Psidium guajava L.) desidratada. In: Congresso Brasileiro De Engenharia Química, p. 1-8.

Moreira, R. R. D., Martins, R.R., Botelho, G. Z., Santos, V. T., Cavaleiro, L. E., Salgueiro, C., Andrade, L., Martins, G. G. \& Henrique, C. (2014). Composition and activity against oral pathogens of the essential oil Melampodium divaricatum (Rich.) DC. Chemistry \& Biodiversity, 11 (3), $438-444$. 
Nascimento, K. F.(2017). Composição química e avaliação biológica de óleo essencial e composto isolado das folhas de Psidium guineense Swartz (Myrtaceae). Dissertação (Mestrado em Biologia Geral/Bioprospecçao) - Fundação Universidade Federal da Grande Dourados, Faculdade de Ciências Biológicas e Ambientais, Dourados - MS, 68 f.

NIST (National Institute of Standards and Technology). (2011). Standard Reference Database 69. Disponível em: https://webbook.nist.gov/chemistry/. Acesso em: 15 dez. 2021

Noumi, E., Merghni, A., Alreshidi, M. M., Haddad, O., Akmadar, G., Martino, L. D., Mastourini, M., Ceylan, O., Snoussi, M., Al-Sieni, A. \& De Feo, V. (2018). Chromobacterium violaceum and Pseudomonas aeruginosa PAO1: Models for Evaluating Anti-Quorum Sensing Activity of Melaleuca alternifolia Essential Oil and Its Main Component Terpinen-4-ol. Molecules, 23 (2672), 1-16.

Oliveira, I. P., Oliveira, L.C., Moura, C.S. F. T., Junior, A. F. L. \& Rosa, S. R. A. (2012). Cultivo da goiabeira: do plantio ao manejo. Revista Faculdade Montes Belos, 5 (4), 145- 149

Oliveira, N. N. S. (2013). Fenologia de genótipos selecionados de goiabeira (psidium guajava l.) e caracterização molecular de acessos de psidium spp. Via marcadores issr. Dissertação (Mestrado em Genética e Melhoramento de Plantas) - Universidade Estadual do Norte Fluminense Darcy Ribeiro - UENF, Centro de Ciências e Tecnologias Agropecuárias, Goytacazes - RJ, 67 f.

Oliveira, J. D., Alves, C.C.F., Miranda, M. L. D., Martins, C. H. G., Silva, T. S., Ambrosio, M. A. L. V., Alves, J. M. \& Silva, J. P. (2016). Rendimento, composição química e atividades antimicrobiana e antioxidante do óleo essencial de folhas de Campomanesia adamantium submetidas a diferentes métodos de secagem. Revista Brasileira de Plantas Medicinais, 18 (2), 502- 510.

Pereira, C. K. B. (2010). Estudo químico e atividades microbiológicas de espécies do gênero Psidium. Dissertação (Mestre em Bioprospeccao Molecular) Universidade Regional do Cariri, Centro de Ciências Biológicas e da Saúde, Departamento de Química Biológica, Crato, CE, 113 f.

Pinho, A. I. (2015). Efeitos biológicos do óleo essencial e do extrato hidro alcóolico das folhas de Psidium guajava var. pomifera L. Tese (Doutorado: Programa de Pós - Graduação em Ciências Biológicas: Bioquímica Toxicológica) - Universidade Federal de Santa Maria, Centro de Ciências Naturais e Exatas, RS, $90 \mathrm{f}$.

Ponzo, F. S. (2014). Tratamento térmico, etanol, quitosana e 1-metilciclopropeno no controle da antracnose em goiabas 'Kumagai'. Tese (Doutorado: programa de Pós - Graduação em Agricultura Tropical e Subtropical) - Instituto Agronômico, Campinas, SP, 114 f.

Rizzo, L. Y. (2011). Atividade anticâncer in vitro e in vivo de Psidium guajava L. (nome popular: goiabeira). Dissertação (Mestrado em Biologia Celular e estrutural) - Universidade Estadual de Campinas, Instituto de Biologia, SP, $100 \mathrm{f}$.

Sá. R. D.; Santana, A. S. C. O. \& Randau, K. P. (2016). Caracterização anatômica e histoquímica das folhas de Eugenia uniflora L. Journal of Environmental Analysis and Progress, 1 (1), 96-105.

Satyal, P., Paudel, P., Lamichhane, B. \& Setzer, W. N. (2015). Leaf essencial oil composition and bioactivity of Psidium guajava from Kathmandu, Nepal. American Journal of Essencial Oils and Natural Products, 2 (2), 11-14.

Saulle, C. C. (2018). Analise morfoanatômicas de folhas e caules e análise química e biológica do óleo essencial de Eucalyptus saligna Sm. (Myrtaceae). Dissertação (Mestrado em Ciências da saúde) - Universidade Estadual de Ponta Grossa, Ponta Grossa, 99 f.

Silva, E. A. J., Silva, V. P., Alves, C. C. F., Alves, J. M., Souchie, E. L. \& Barbosa, L. C. A. (2016). Harvest time on the content and chemical composition of essential oil from leaves of guava. Ciência rural, 46 (10), 1771- 1776.

Silva, E. A. J. (2019). Óleo essencial das folhas de Psidium guajava: Controle de Sclerotinia sclerotiorum em soja, atividade de bactericida e anticariogênica. Tese (Doutorado: Pós-Graduação em Ciências Agrárias) - Instituto Federal de Educação, Ciência e Tecnologia Goiano, Rio verde, 71 f.

Silva, E.A.J., Silva, V. P., Alves, C. C. F., Alves, J. M., Souchie, E. L. \& Barbosa, L. C. A. (2018). Chemical composition of the essential oil of Psidium guajava leaves and its toxicity against. Sclerotinia sclerotiorum. Semina: Ciências Agrárias, 39 (2), 865-874.

Souza, T. S. (2015). Perfil cromatográfico do óleo essencial e diversidade quimiotípica de Psidium guajava L. Tese (Doutorado: Pós-Graduação em Produção Vegetal) - Universidade Federal do Espirito Santo, Centro de Ciências Agrárias, Alegre- ES, $97 \mathrm{f}$.

Souza, T. S., Ferreira, M. F. S., Menini, J., Souza, J. R. C. L., Bernardes, C. O. \& Ferreira, A.(2018). Chemotype diversity of Psidium guajava L. Phytochemistry, 153, 129-137.

Torres, A.; Turco, C. P.; \& Pedreca, P. (2005). Perspectivas da goiaba: o Brasil maior produtor da espécie vermelha. Revista agronegócios da FGV, 24p. 